\title{
In-Phase Coupling Forces Induced by Air Flow and Drive Force in Split-Mode Silicon Micromachined Linear Vibratory Gyroscopes
}

\author{
Wenwen Zhou, Bin Zhou, Zhiyong Chen and Rong Zhang* \\ Department of Precision Instruments and Mechanology, Tsinghua University, \\ Beijing 100084, P. R. China
}

(Received March 1, 2010; accepted June 17, 2010)

Key words: coupling forces, air damping, vacuum package, vibratory microgyroscopes

In this paper, we report on the sources, variations and identification of in-phase coupling forces induced by air flow and drive force in split-mode silicon micromachined linear vibratory gyroscopes. Air damping coupling force and drive coupling force are two main components of in-phase coupling forces, derived from air flow and fabrication imperfections, respectively. The expression of in-phase coupling forces induced by these two factors is given and their existence is demonstrated by a novel coupling identification method. Experimental data indicate that air damping coupling is the dominant coupling force in in-phase coupling. The sensitivity of in-phase coupling to vacuum will be a theoretical instruction to packaging requirements and 1 Torr is an optimum vacuum not requiring complex packaging technology, but restraining in-phase coupling forces effectively.

\section{Introduction}

Micromachined rate gyroscopes are becoming indispensable as key elements for aerospace applications, vehicle dynamic control systems, and consumer electronics industries. Successful integration of microgyroscope systems has already been demonstrated in a host of consumer applications such as gaming consoles, image stabilization in digital cameras, and traction control systems in automobiles. ${ }^{(1,2)}$ However, driven by high-volume commercial market needs, applications continue to grow for highprecision micromachined gyroscopes. Hence, there is an inevitable need for improved device performance. ${ }^{(3)}$

At present, the challenge is to obtain excellent performance in a microgyroscope where cross-coupling forces exist. Obstacles include manufacturing tolerances and the relatively large magnitudes of non-Coriolis forces. ${ }^{(4)}$ Coupling forces can be classified

${ }^{*}$ Corresponding author: e-mail: 520zww@gmail.com 
into in-phase coupling forces and quadrature coupling forces on the basis of their phase angle relationship. Some coupling errors are demonstrated through the model ${ }^{(5)}$ proposed in ref. 5, and cross-coupling effects due to nonideal behavior as well as the effect on the signal were modeled. The nonideal behavior of the driving unit is proved to be the determining factor for the quadrature signal..$^{(6-10)}$

Errors caused by quadrature coupling forces are greatly reduced at the DC output by demodulation function, while the extra in-phase coupling forces appear directly as DC bias errors. In this paper, the authors report on the mechanism of in-phase coupling forces induced by air flow and drive force in a split-mode silicon micromachined linear vibratory gyroscope, and the variations of in-phase coupling forces are observed by experiments based on a novel identification method. The theory and sources of coupling forces are discussed in $\S 2$. In $\S 3$, we describe the method of identifying in-phase coupling forces. Experimental results involved in relative discussion are the subjects of $\S 4$. In $\S 5$, we summarize the results of this work.

\section{Theory}

Micromachined vibratory gyroscopes are based on the Coriolis-induced transfer of energy between two vibration modes of a microstructure. They can operate in either mode-matched or split-mode condition. Under mode-matched condition, the sense mode is designed to have the same (or nearly the same) resonant frequency as the drive mode. Hence, the rotation-induced Coriolis signal is amplified by the mechanical quality factor of the sense mode. In split-mode condition, the drive mode and the sense mode are separated in resonant frequency. The sense mode is then a controlled mode that operates in a similar manner to an accelerometer and measures the Coriolis acceleration. ${ }^{(11)}$

A linear vibratory gyroscope structure, as shown in Fig. 1, is comprised of a proof mass, supported by several pairs of flexural springs, and anchored at four square posts. The proof mass is driven in-plane into resonance along the drive axis using electrostatic drive combs, which are symmetrically distributed to the proof mass. An input rotation signal normal to the device plane causes a Coriolis-induced transfer of energy to the sense vibration mode. The resulting in-plane displacement of the sense axis is sensed capacitively at sense combs located along the sense axis. For closed-loop control of the drive mode, the displacement of the drive axis is also sensed capacitively at drive monitor combs, which are located next to drive combs. Sense combs and balance combs have the same shape, but different functions. Balance combs are used to apply forces along the sense axis, when the sense mode also needs to be under close-loop control.

With only linear terms considered, the drive axis and the sense axis dynamics are described by second-order spring-mass systems with coupling between modes

$$
\begin{aligned}
& \text { Drive: } F_{\mathrm{d}}(t)=m x^{\prime \prime}(t)+b_{\mathrm{d}} x^{\prime}(t)+k_{\mathrm{d}} x(t)+\left(b_{\mathrm{sd}}-2 m \Omega_{\mathrm{I}}\right) y^{\prime}(t)+k_{\mathrm{sd}} y(t), \\
& \text { Sense: } \alpha F_{\mathrm{d}}(t)=m y^{\prime \prime}(t)+b_{\mathrm{s}} y^{\prime}(t)+k_{\mathrm{s}} y(t)+\left(b_{\mathrm{ds}}+2 m \Omega_{\mathrm{I}}\right) x^{\prime}(t)+k_{\mathrm{ds}} x(t),
\end{aligned}
$$




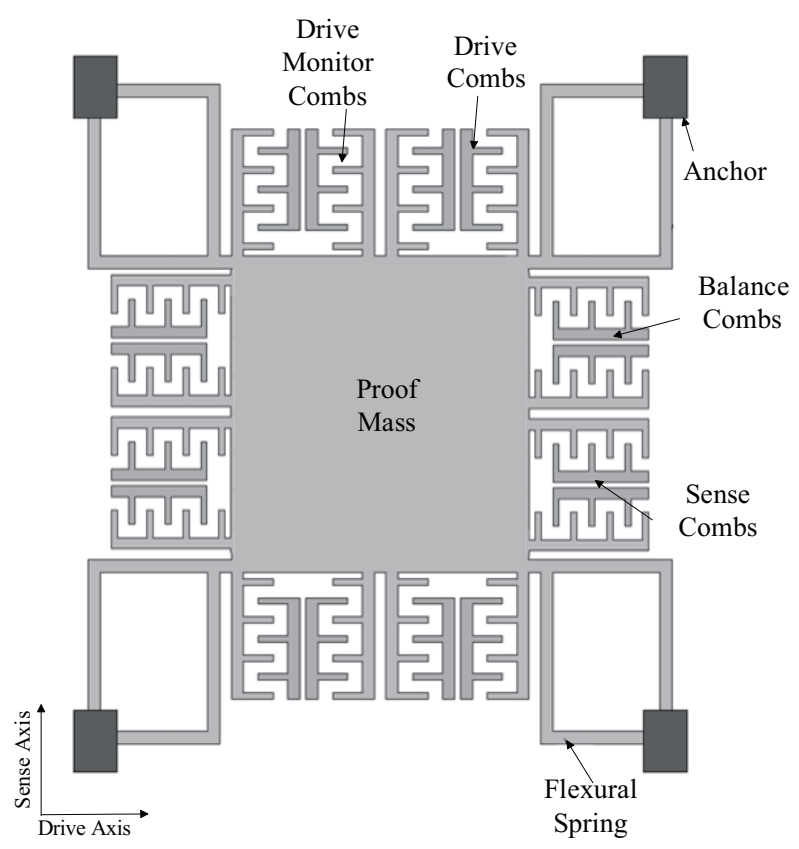

Fig. 1. Structural overview of a split-mode silicon micromachined linear vibratory gyroscope.

where

$F_{\mathrm{d}}(t) \quad$ drive force applied by drive combs in Fig. 1;

$m \quad$ mass of the proof;

$b_{\mathrm{d}}, b_{\mathrm{s}} \quad$ damping in the drive axis and the sense axis, respectively;

$k_{\mathrm{d}}, k_{\mathrm{s}} \quad$ stiffness in the drive axis and the sense axis, respectively;

$k_{\mathrm{ds}} \quad$ quadrature coupling from the drive axis into the sense axis;

$k_{\mathrm{sd}} \quad$ quadrature coupling from the sense axis into the drive axis;

$b_{\mathrm{ds}} \quad$ in-phase coupling from the drive axis into the sense axis;

$b_{\text {sd }} \quad$ in-phase coupling from the sense axis into the drive axis;

$x(t) \quad$ motion along the drive axis;

$y(t) \quad$ motion along the sense axis;

$\Omega_{\mathrm{I}} \quad$ slowly varying input rate;

$\alpha \quad$ drive force coupling to the sense axis.

A fourth-order coupling system transfer function from drive force to sense motion can be obtained based on eqs. (1) and (2):

$$
\frac{Y(s)}{F_{\mathrm{d}}(s)}=\frac{\alpha\left(m s^{2}+b_{\mathrm{d}} s+k_{\mathrm{d}}\right)-\left(b_{\mathrm{ds}}+2 m \Omega_{\mathrm{I}}\right) s-k_{\mathrm{ds}}}{\left(m s^{2}+b_{\mathrm{d}} s+k_{\mathrm{d}}\right)\left(m s^{2}+b_{\mathrm{s}} s+k_{\mathrm{s}}\right)-\left[\left(b_{\mathrm{ds}}-2 m \Omega_{\mathrm{I}}\right) s+k_{\mathrm{sd}}\right]\left[\left(b_{\mathrm{ds}}+2 m \Omega_{\mathrm{I}}\right) s+k_{\mathrm{ds}}\right]} .
$$


Coupling forces from the drive mode to the sense mode can be classified into two types: quadrature coupling and in-phase coupling, depending upon the phase relationship with Coriolis force. A $90^{\circ}$ phase lag exists between quadrature coupling forces and Coriolis force, such as stiffness coupling force $\left(k_{\mathrm{ds}} x(t)\right.$ shown in eq. (2)), which is a result of fabrication imperfection or environmental variety (temperature variety). In-phase coupling forces, on the other hand, have the same phase with Coriolis force. Because of this phase difference, as mentioned in $\S 1$, quadrature forces can be greatly reduced by demodulation, while in-phase forces appear as bias errors. Hence, in-phase coupling forces directly determine the gyro bias stability, which is a key performance parameter of vibratory gyroscopes. In-phase coupling forces are themselves further classified into several types depending upon their source, and air damping coupling force and drive coupling force $\left(\alpha F_{\mathrm{d}}(t)\right.$ shown in eq. (2)) are two main components.

Air damping force, including the squeeze film and the slide film damping, is derived from the variety of air fluid surrounding the structure in a package shell.(12) Air damping coupling is one component of in-phase coupling, as Coriolis force $F_{\mathrm{c}}=2 m \Omega_{\mathrm{I}} \times x^{\prime}(t)$ and air damping coupling force $F_{\mathrm{air}}=b_{\mathrm{ds}} x^{\prime}(t)$ are both proportional to velocity $x^{\prime}(t)$. Ignoring the effects of combs and beams, Fig. 2 shows the vibration of the proof mass along the drive axis and the motion of air flow between the proof mass and the shell, on the condition that two modes were fabricated exactly orthogonal. Axis $\mathrm{y}$ is the sense axis and axis $\mathrm{x}$ is the drive axis, in which the gyroscope vibrates with the velocity $x^{\prime}(t)$. Cross coupling between the drive mode and the sense mode will not appear when there is no fabrication error at all. In this situation, only the drive mode can be affected by air damping force, which is comprised of squeeze film damping between the proof mass and side walls along axis y, slide film I damping between the proof mass and top (bottom) wall, and slide film II damping between the proof mass and side walls along axis $\mathrm{x}$.

Figure 3 describes the air flow induced by the vibration of the proof mass along the drive axis in the presence of fabrication imperfections. The squeeze film damping along

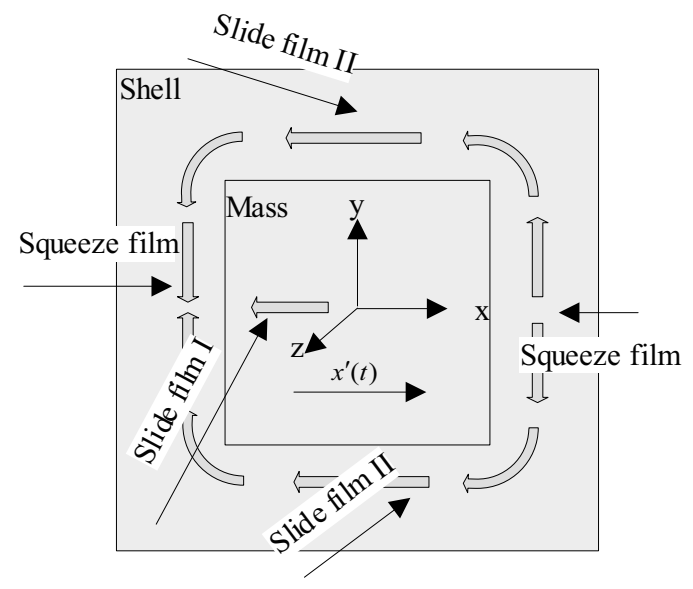

Fig. 2. Air flow induced by the proof-mass motion in ideal fabrication. 


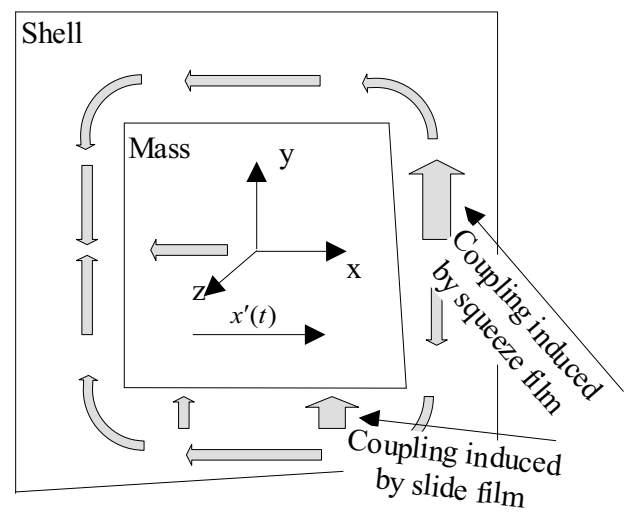

Fig. 3. Air damping coupling caused by fabrication imperfections (coupling has been exaggerated for clarity).

the drive axis creates a coupling force along the sense axis when the nonorthogonality of two axes appears. This kind of nonorthogonality comes from mass fabrication, shell fabrication or assembly error.

All the pressures to the mass proof along the sense axis are equal, as the air-flow channel in Fig. 1 is symmetrical. Thus, there is no force coupling to the sense axis, while in Fig. 3, a coupling force induced by slide film damping has emerged owing to the imperfection of the air-flow channel. Hence, coupling forces induced by air damping appear in the sense mode.

The frequency response of the undesired air damping coupling force $F_{\text {air }}$ can be calculated from:

$$
F_{\mathrm{air}}(j \omega)=j b_{\mathrm{ds}} \omega \cdot x(j \omega)
$$

Since $b_{\mathrm{ds}}$ is changed by the air-flow channel and air molecules motion, the bias output induced by air damping coupling is sensitive to vacuum or temperature variations.

Drive coupling force is another component of in-phase coupling forces, which is derived from comb imperfections, as shown in Fig. 4. Sense electrodes can detect drive force directly in the sense mode because of askew drive combs. Electrical coupling from the drive electrode to the sense electrode can also be defined as a kind of drive coupling force. As the drive axis dynamics is a second-order spring-mass system and the drive mode operates at its resonant frequency, drive force is in quadrature with the proof-mass position, i.e., in phase with the proof mass velocity $x^{\prime}(t)$. Thus, drive coupling force belongs to in-phase coupling forces. The frequency response of drive force $F_{\mathrm{d}}$ can be expressed by

$$
F_{\mathrm{d}}(j \omega)=m\left(\omega_{\text {nd }}^{2}-\omega^{2}+j \omega_{\text {nd }} \omega / Q_{\mathrm{d}}\right) x(j \omega),
$$




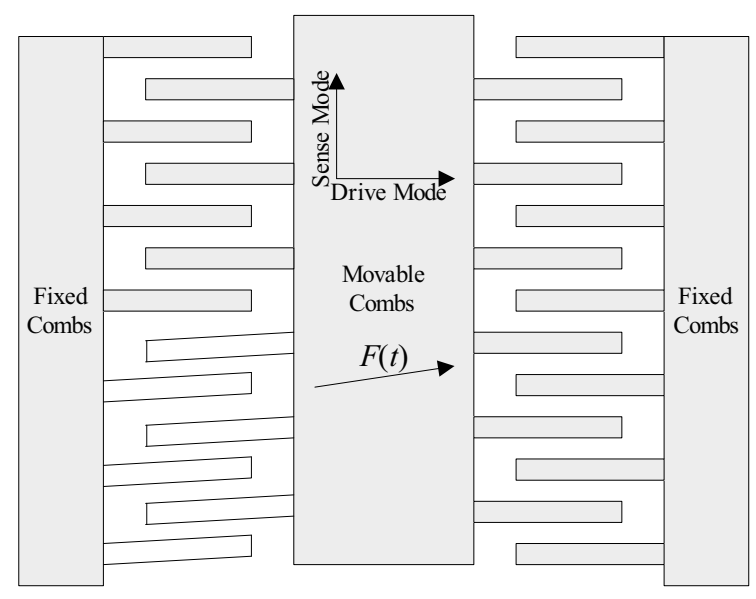

Fig. 4. Drive coupling force caused by fabrication imperfections (coupling has been exaggerated for clarity).

where $\omega_{\text {nd }}$ and $Q_{\mathrm{d}}$ are the natural frequency and quality factor of the drive axis, respectively.

As the natural frequency and the resonant frequency are almost equal when the quality factor is sufficiently high, drive coupling force at resonant frequency can be calculated by

$$
\alpha F_{\mathrm{d}}\left(j \omega_{\mathrm{nd}}\right)=\frac{j \alpha \omega_{\mathrm{nd}}^{2}}{Q_{\mathrm{d}}} x\left(j \omega_{\mathrm{nd}}\right)
$$

In eq. (2), drive coupling force is defined as an external excitation, while air damping coupling is defined as an internal coupling. Thus, a $180^{\circ}$ phase lag exists between them and a minus will be induced. The equation of in-phase coupling forces induced by air flow and drive force can be obtained from eqs. (4) and (6):

$$
F_{\text {in }}\left(j \omega_{\text {nd }}\right)=j\left(b_{\mathrm{ds}} \omega_{\text {nd }}-\frac{\alpha m \omega_{\text {nd }}^{2}}{Q_{\mathrm{d}}}\right) x\left(j \omega_{\text {nd }}\right) .
$$

\section{Identification of In-Phase Coupling Forces}

As the intercoupling of micromachined gyroscopes is extraordinary complicated, it is difficult to measure or observe each coupling force individually under the present experimental conditions. Hence, it is not possible to analyze each coupling force separately for further research. However, the ratio of in-phase to quadrature coupling forces can be measured by a novel identification method described in this section, and it is possible to observe the variations of in-phase coupling forces if maintaining quadrature coupling forces invariant. The coupling forces ratio is calculated from 


$$
\left|\frac{F_{\text {in }}\left(j \omega_{\text {nd }}\right)}{F_{\mathrm{q}}\left(j \omega_{\text {nd }}\right)}\right|=\left|\frac{j\left(b_{\mathrm{ds}} \omega_{\text {nd }}-\alpha m \omega_{\text {nd }}^{2} / Q_{\mathrm{d}}\right) x\left(j \omega_{\mathrm{nd}}\right)}{k_{\mathrm{ds}} x\left(j \omega_{\mathrm{nd}}\right)}\right|=\left|\frac{b_{\mathrm{ds}} \omega_{\mathrm{nd}}-\alpha m \omega_{\mathrm{nd}}^{2} / Q_{\mathrm{d}}}{k_{\mathrm{ds}}}\right| .
$$

Since the vibrating of the drive mode is the dominant motion, coupling forces from the sense mode to the drive mode can be neglected compared with those forces from the drive mode to the sense mode when the gyroscope operates at the natural frequency of the drive axis. Therefore, in eq. (3), both $k_{\mathrm{sd}}=0$ and $b_{\mathrm{sd}}=0$. The fourth-order coupling system can be further simplified without the presence of angle rate input, and its frequency response can be expressed by

$$
\frac{Y(j \omega)}{F_{\mathrm{d}}(j \omega)}=\frac{\left(\alpha m \omega_{\mathrm{nd}}^{2}-\alpha m \omega^{2}-k_{\mathrm{ds}}\right)+j\left(\alpha m \omega_{\mathrm{nd}} \omega / Q_{\mathrm{d}}-b_{\mathrm{ds}} \omega\right)}{m^{2}\left(\omega_{\mathrm{nd}}^{2}-\omega^{2}+j \omega_{\mathrm{nd}} \omega / Q_{\mathrm{d}}\right)\left(\omega_{\mathrm{ns}}^{2}-\omega^{2}+j \omega_{\mathrm{ns}} \omega / Q_{\mathrm{s}}\right)},
$$

where $\omega_{\mathrm{ns}}$ and $Q_{\mathrm{s}}$ are the natural frequency and quality factor of the sense axis, respectively.

The phase-frequency response of eq. (9) is calculated from

$$
\varphi_{\mathrm{ds}}(j \omega)=\varphi_{\mathrm{d}}(j \omega)+\varphi_{\mathrm{s}}(j \omega)+\varphi_{\text {ratio }}(j \omega),
$$

where

$\varphi_{\mathrm{ds}}(j \omega)$ the fourth-order phase from drive force to sense motion;

$\varphi_{\mathrm{d}}(j \omega)$ the second-order phase from drive force to drive motion;

$\varphi_{\mathrm{s}}(j \omega)$ the second-order phase from sense force to sense motion;

$\varphi_{\text {ratio }}(j \omega)$ the phase of $\left[\left(\alpha m \omega_{\text {nd }}^{2}-\alpha m \omega^{2}-k_{\mathrm{ds}}\right)+j\left(\alpha m \omega_{\text {nd }} \omega / Q_{\mathrm{d}}-b_{\mathrm{ds}} \omega\right)\right]$.

At the resonant frequency, $\left|\tan \left[\varphi_{\text {ratio }}\left(j \omega_{\text {nd }}\right)\right]\right|$ is the ratio of in-phase to quadrature coupling forces:

$$
\left|\tan \left[\varphi_{\text {ratio }}\left(j \omega_{\text {nd }}\right)\right]\right|=\left|\frac{b_{\mathrm{ds}} \omega_{\text {nd }}-\alpha m \omega_{\text {nd }}^{2} / Q_{\mathrm{d}}}{k_{\mathrm{ds}}}\right|=\left|\frac{F_{\text {in }}\left(j \omega_{\text {nd }}\right)}{F_{\mathrm{q}}\left(j \omega_{\text {nd }}\right)}\right| .
$$

According to eq. (11), the coupling forces ratio will be calculated after open-loop frequency-sweep tests on the fourth-order drive-sense system, the second-order driveaxis system, and the second-order sense-axis system, obtaining $\varphi_{\mathrm{ds}}(j \omega), \varphi_{\mathrm{d}}(j \omega)$ and $\varphi_{\mathrm{s}}(j \omega)$, respectively. On the basis of this, in-phase coupling forces can be identified from the system, when no other factors changing quadrature coupling are involved.

\section{Results and Discussion}

To extract the detailed motion information from a split-mode micro-electromechanical systems (MEMS) linear vibratory gyroscope, a digital signal readout system is required. The signal processing and readout block diagram, shown in Fig. 5, have been implemented using a preamplifier circuit board and the NI-PCI-4461 24-bit dynamic signal acquisition board. 


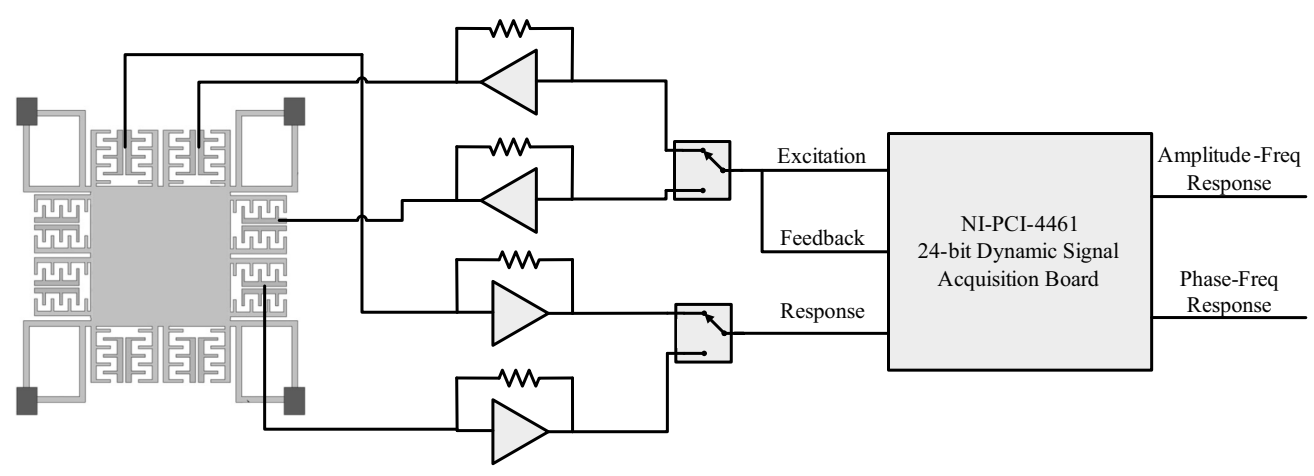

Fig. 5. Schematic of the readout circuitry for identifying coupling forces.

As the purpose of this paper is to analyze in-phase coupling forces induced by air flow and drive force, we change the experimental condition to affect these forces. Changing air pressure is an ideal method to affect in-phase coupling forces, while maintaining quadrature coupling forces constant. Theoretically, the existence of in-phase coupling can be demonstrated and its variations will be observed in different vacuums. However, the quality factors of both the drive mode and the sense mode will become high once the air pressure decreases. If the gyroscope is excited by a constant drive voltage along the drive axis during air pressure decrease, the change of quality factor in the drive mode will cause differences in the amplitude of the displacement along this axis, and many other influencing factors will be involved in the experimental result. The same situation will appear in the sense mode when the input signal is applied to the sense axis. Thus, the amplitude of the displacement should be kept constant by changing the drive voltage in different vacuums.

The devices used in this study were split-mode silicon MEMS linear vibratory gyroscopes designed by Tsinghua University, as shown in Fig. 1. All the tests in this chapter are open-loop frequency-sweep tests. The 0908\# gyroscope was tested in various vacuums, ranging from 1 atmosphere to 300 mTorr. Three frequency-sweep tests were implemented in every vacuum, including the drive axis input/the sense axis output, to obtain $\varphi_{\mathrm{ds}}(j \omega)$; the drive axis input/the drive axis output, to obtain $\varphi_{\mathrm{d}}(j \omega)$; the sense axis input/the sense axis output, to obtain $\varphi_{\mathrm{s}}(j \omega)$. Some characters of the 0908\# gyroscope in different vacuums are shown in Table 1 .

When the tests were the drive axis input (to obtain $\varphi_{\mathrm{ds}}(j \omega)$ and $\varphi_{\mathrm{d}}(j \omega)$ ), the amplitude of the displacement along the drive axis was kept constant in different vacuums by changing the input voltage in the drive axis according to Table 1 . When the tests were the sense axis input (to obtain $\varphi_{\mathrm{s}}(j \omega)$ ), the amplitude of the displacement along the sense axis was also kept constant by changing the input voltage in the sense axis. Therefore, during the vacuum variation, only two changed factors affect the gyroscope: air flow and drive force.

As the natural frequency of the drive axis will be changed by temperature, we usually analyze the data within $40 \mathrm{~Hz}$ around it. The data of $\varphi_{\mathrm{ds}}(j \omega), \varphi_{\mathrm{d}}(j \omega)$ and $\varphi_{\mathrm{s}}(j \omega)$ at each 
Table 1

Characteristics of the 0908\# gyroscope in different vacuums.

\begin{tabular}{lccccc}
\hline & $1 \mathrm{~atm}$. & 25 Torr & 5 Torr & 1 Torr & 300 mTorr \\
\hline Quality factor in the drive mode & 185.66 & 351.02 & 1008.9 & 2552.6 & 3609.6 \\
Input voltage in the drive axis (V) & 2.00 & 1.06 & 0.368 & 0.145 & 0.103 \\
Amplitude of displacement & 0.401 & 0.400 & 0.400 & 0.401 & 0.400 \\
$\quad$ along the drive axis $(x(j \omega))(\mathrm{V})$ & 79.292 & 178.8 & 657.31 & 2473.6 & 4525.6 \\
Quality factor in the sense mode & 4.38 & 2.02 & 0.534 & 0.147 & 0.0776 \\
$\begin{array}{l}\text { Input voltage in the sense axis }(\mathrm{V}) \\
\text { Amplitude of displacement }\end{array}$ & 0.400 & 0.400 & 0.400 & 0.401 & 0.400 \\
along the sense axis $(y(j \omega))(\mathrm{V})$ & & & & & \\
\hline
\end{tabular}

vacuum point were obtained, and the curves in 1 atmosphere and 300 mTorr are plotted in Figs. 6 and 7.

The phase lag between $\varphi_{\mathrm{ds}}(j \omega)$ and $\varphi_{\mathrm{d}}(j \omega)$ is comprised of $\varphi_{\mathrm{s}}(j \omega)$ and $\varphi_{\text {ratio }}(j \omega)$, and it was obvious in 1 atmosphere. However, the phase lag almost disappeared in 300 mTorr, because the $\varphi_{\mathrm{s}}(j \omega)$ decreased by a increased quality factor in the sense mode, and the $\varphi_{\text {ratio }}(j \omega)$ decreased by the reduction of in-phase coupling forces. The phase curves between 1 atmosphere and $300 \mathrm{mTorr}$ are not given, as the coupling forces ratio can reflect the same information directly. The in-phase to quadrature coupling forces ratio of the 0908\# gyroscope calculated from eqs. (11) is plotted in Fig. 8.

The sensitivity of the coupling forces ratio $\left|F_{\text {in }}(j \omega) / F_{\mathrm{q}}(j \omega)\right|$ to vacuum can be observed in Fig. 8. Since the quadrature coupling forces are unchanged, these coupling forces ratio curves can reflect in-phase coupling variations. When the gyroscope was operated under high-vacuum condition, in-phase coupling forces from eq. (7) decreased. This phenomenon was derived from in-phase coupling's two components: air damping coupling force and drive coupling force.

As described in the theory, there are many components in in-phase coupling forces. However, only the coupling forces induced by air flow and drive force were changed during the vacuum variation. The coupling force induced by air flow, i.e., air damping coupling, was reduced with the decrease in $b_{\mathrm{ds}}$, as there are less air molecules in a higher vacuum. In addition to the lower air damping coupling, high vacuum also provided a high quality factor. Thus, the drive force $F_{\mathrm{d}}(j \omega)$ to the drive axis should be reduced from 2 to $0.103 \mathrm{~V}$ to maintain a constant amplitude of $x(j \omega)$ when obtaining $\varphi_{\mathrm{ds}}(j \omega)$ and $\varphi_{\mathrm{d}}(j \omega)$. As a result, the coupling force induced by drive force, i.e., drive coupling force $\alpha F_{\mathrm{d}}(j \omega)$ also decreased. In this situation, electrical coupling from the driving electrode to the sense electrode may also be changed. To clarify the dominant part of in-phase coupling forces during the vacuum variation, further tests have been implemented on the 0908\# gyroscope.

We repeated all the tests described above on the 0908\# gyroscope in 1 atmosphere. Drive forces were changed according to Table 1. In these tests, the changed factor affecting in-phase coupling is only the drive coupling force (electrical coupling from the driving electrode to the sense electrode has been defined as a kind of drive coupling force in $\S 2$ ). The coupling forces ratio $\left|F_{\text {in }}(j \omega) / F_{\mathrm{q}}(j \omega)\right|$ is shown in Fig. 9. Compared with Fig. 8, 

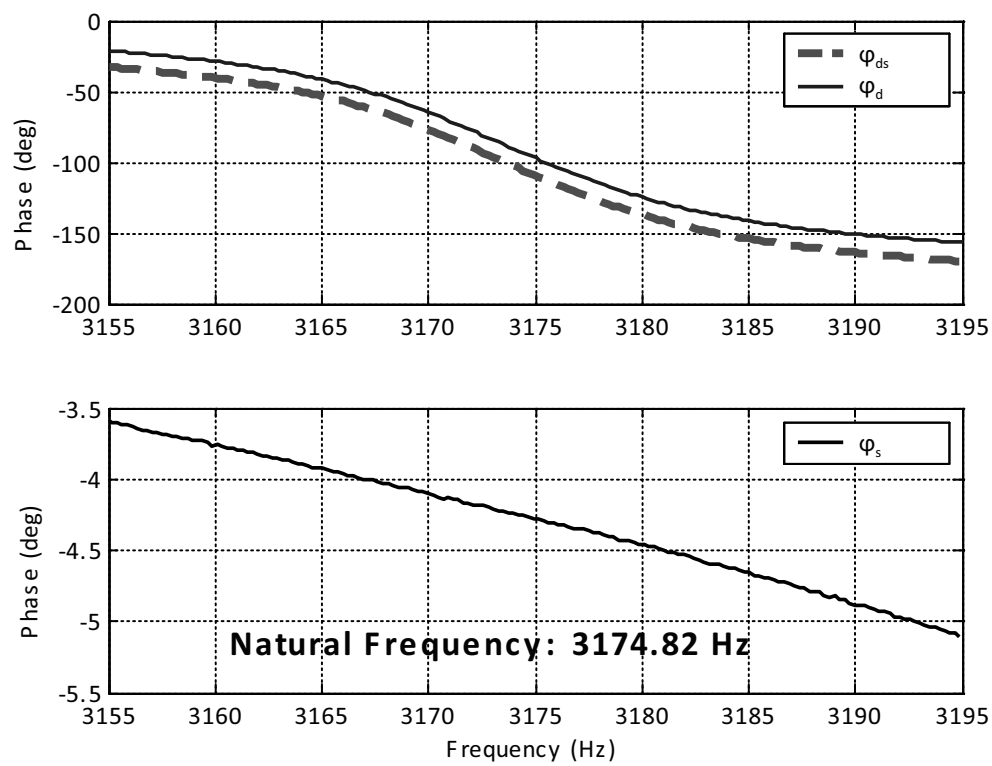

Fig. 6. $\varphi_{\mathrm{ds}}(j \omega), \varphi_{\mathrm{d}}(j \omega)$ and $\varphi_{\mathrm{s}}(j \omega)$ in 1 atmosphere.
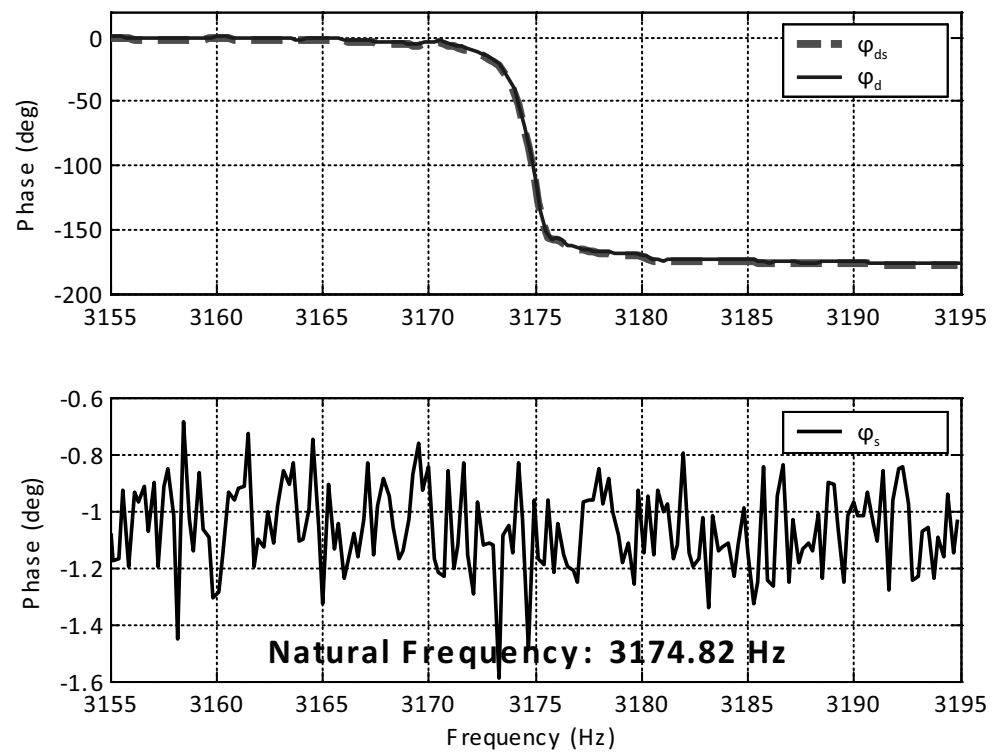

Fig. 7. $\varphi_{\mathrm{ds}}(j \omega), \varphi_{\mathrm{d}}(j \omega)$ and $\varphi_{\mathrm{s}}(j \omega)$ in $300 \mathrm{mTorr}$. 


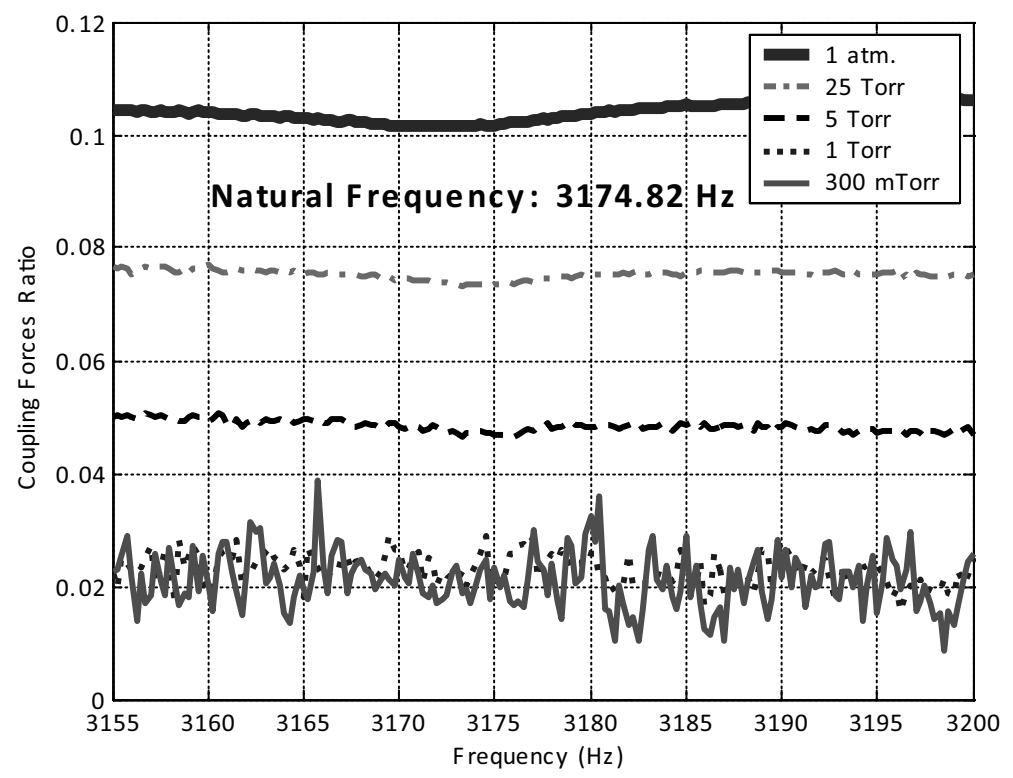

Fig. 8. Coupling forces ratio of the 0908\# gyroscope in different vacuums.

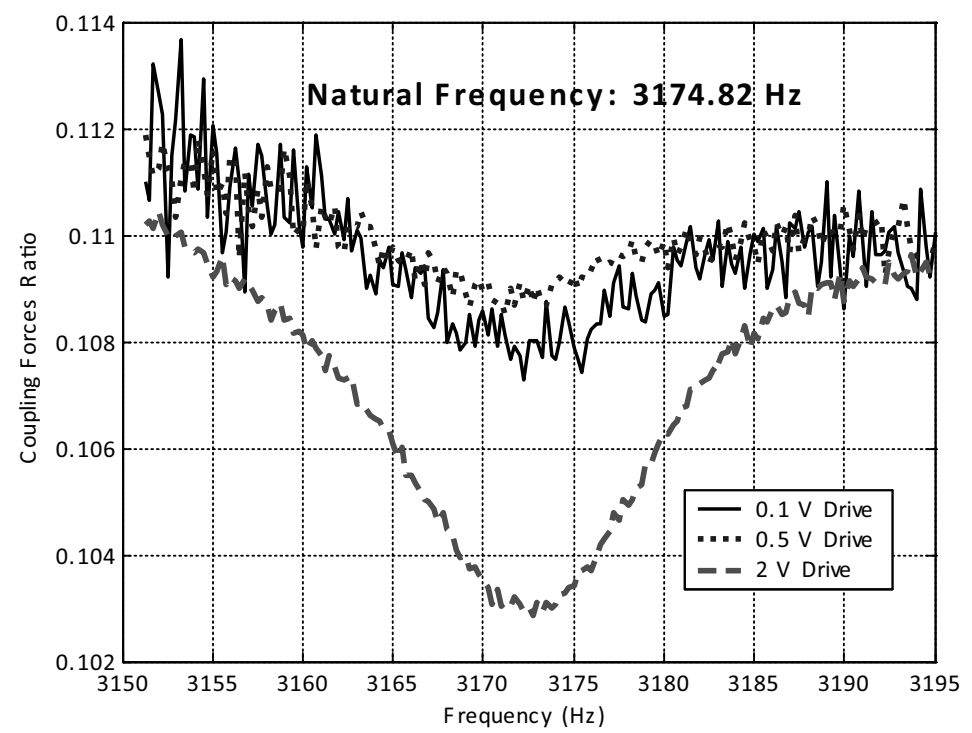

Fig. 9. Coupling forces ratio of $0908 \#$ gyroscope affected by drive coupling force. 
the variations in the coupling forces ratio are obviously small under constant air pressure, although drive coupling force changed markedly. Accordingly, we can conclude that the effect of drive force and electrical coupling between electrodes on in-phase coupling forces is limited, and air damping coupling is the dominant force in in-phase coupling.

The result of the 0901\# gyroscope is shown in Fig. 10, and similar changes were observed in in-phase coupling. The test results of all eight gyroscopes are summarized in Table 2, which show that the reduction of in-phase coupling forces was not obvious when the vacuum was 1 Torr or higher. Even if a vacuum package is not required in a silicon MEMS gyroscope, a low-pressure package is necessary to improve its performance. This result reveals that 1 Torr is an optimum vacuum not requiring complex packaging technology, but restraining in-phase coupling forces effectively.

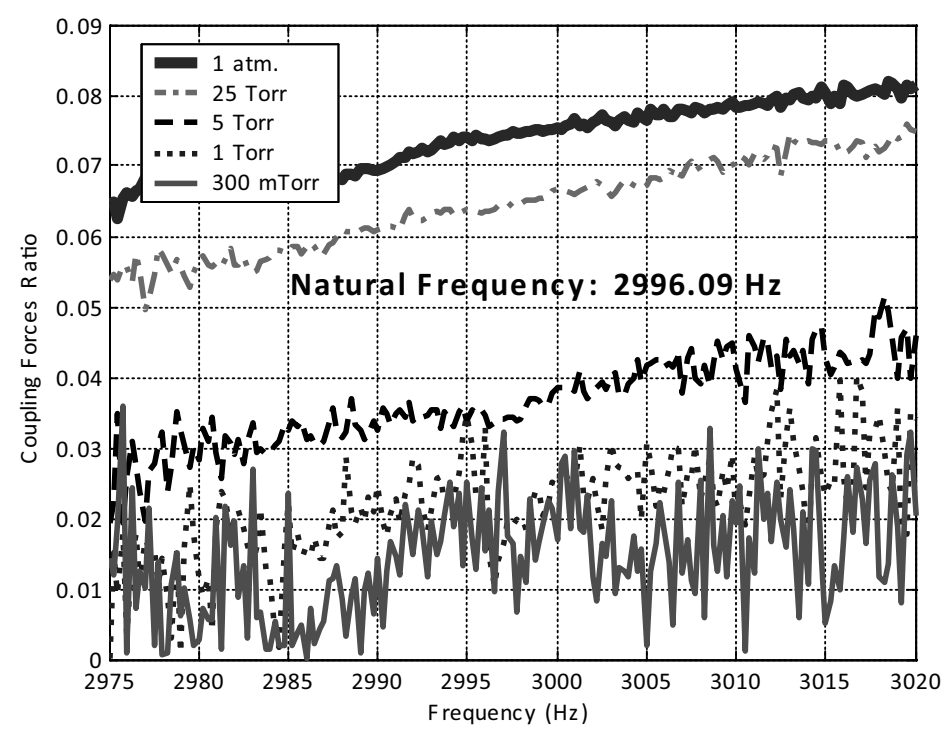

Fig. 10. Coupling forces ratio of $0901 \#$ gyroscope in different vacuums.

Table 2

Coupling forces ratio of eight gyroscope samples in different vacuums.

\begin{tabular}{lccccc}
\hline Gyro sample & 1 atm. & 25 Torr & 5 Torr & 1 Torr & 300 mTorr \\
\hline $0901 \#$ & 0.072 & 0.063 & 0.035 & 0.022 & 0.020 \\
$0902 \#$ & 0.251 & 0.223 & 0.085 & 0.041 & 0.035 \\
$0903 \#$ & 0.103 & 0.083 & 0.042 & 0.026 & 0.023 \\
$0904 \#$ & 0.175 & 0.076 & 0.033 & 0.028 & 0.027 \\
$0905 \#$ & 0.124 & 0.085 & 0.045 & 0.024 & 0.021 \\
$0906 \#$ & 0.085 & 0.061 & 0.042 & 0.035 & 0.032 \\
$0907 \#$ & 0.075 & 0.060 & 0.033 & 0.026 & 0.025 \\
$0908 \#$ & 0.102 & 0.074 & 0.047 & 0.021 & 0.021 \\
\hline
\end{tabular}




\section{Conclusions}

In this paper, we have presented the sources, variations and identification of in-phase coupling forces induced by air flow and drive force in split-mode silicon micromachined linear vibratory gyroscopes. The existence of in-phase coupling forces induced by these two factors was demonstrated and their variations were observed by a novel coupling forces identification method. Air damping coupling is the dominant coupling force in in-phase coupling, as experimental results showed the variations caused by it were approximately ten times higher than those caused by drive force coupling. Further tests on all eight gyroscope samples indicated that in-phase coupling forces were less sensitive to air pressure when the vacuum was 1 Torr or higher. This will be a theoretical instruction to packaging requirements and will permit this type of gyroscope to approach an ideal performance in the near future.

\section{Acknowledgment}

This work was partially supported by the National High-tech Program of China (863 Program).

\section{References}

1 J. Marek: Proc. IEEE MEMS 2007 (IEEE, Kobe, 2007) p. 59.

2 B. Vigna: Proc. IEEE Thermal, Mech, Multiphys, Simulation and Experiments in MEMS 2006 (IEEE, Como, 2006) p. 1.

3 M. F. Zaman, A. Sharma and F. Ayazi: IEEE Sens. J. 9 (2009) 616.

4 M. S. Weinberg and A. Kourepenis: J. MEMS. 15 (2006) 479.

5 E. Kanso, A. J. Szeri and A. P. Pisano: J. MEMS. 13 (2004) 323.

6 M. Braxmaier, A. Gaiber, T. Link, A. Schumacher, I. Simon, J. Frech, H. Sandmaier and W. Lang: Proc. IEEE Transducers 2003 (IEEE, Boston, 2003) p. 167.

7 C. C. Painter and A. M. Shkel: IEEE Sens. J. 3 (2003) 595.

8 C. Acar and A. M. Shkel: IEEE Sens. J. 3 (2003) 497.

9 Z. C. Feng and K. Gore: IEEE Sens. J. 4 (2004) 80.

10 C. Acar and A. M. Shkel: J. Micromech. Microeng. 13 (2003) 229.

11 M. F. Zaman, A. Sharma, Z. Hao and F. Ayazi: J. MEMS. 17 (2008) 1526.

12 M. Bao: Micro Mechanical Transducers-Pressure Sensors, Accelerometer and Gyroscopes (Elsevier, Amsterdam, 2000) Chap. 3. 